\title{
Genetic Analysis of Heterotic Crosses in Rice (Oryza sativa L.) under Rainfed Ecosystem
}

\author{
Madhuri Arya ${ }^{1}$, Aradhana Singh ${ }^{3}$, H. C. Singh ${ }^{2}$, Shama Parveen ${ }^{3}$, Manoj Kumar ${ }^{4}$, Mukh Ram ${ }^{3}$ and Shipra Shalini ${ }^{1 *}$ \\ ${ }^{1}$ Dept. of Plant Breeding and Genetics, RAC, BAU, Ranchi, Jharkhand (834 001), India \\ ${ }^{2}$ Dept. of Genetics and Plant Breeding, CSAUA\&T, Kanpur, UP (208 002), India \\ ${ }^{3}$ Dept. of Genetics and Plant Breeding, BHU, Varanasi, UP (221 005), India \\ ${ }^{4}$ Dept. of Crop physiology, BAU, Ranchi, Jharkhand (834 006), India
}

\section{Article History}

Manuscript No. AR813b

Received in $25^{\text {th }}$ June, 2014

Received in revised form $5^{\text {th }}$ December, 2014

Accepted in final form $25^{\text {th }}$ January, 2015

\section{Correspondence to}

${ }^{*}$ E-mail: shalinishipra76@gmail.com

\section{Keywords}

Gene effects, heterosis, heterobeltiosis, rice, yield

\begin{abstract}
An experiment consisting of fifteen testers, three lines and their forty five crosses was conducted at crop research farm of Birsa Agriculture University, Kanke, Ranchi, Jharkhand during kharif season. Each parent and $\mathrm{F}_{1} \mathrm{~s}$ were sown in five rows plot of $5.0 \times 1.0 \mathrm{~m}^{2}$ spaced at $20 \times 15 \mathrm{~cm}^{2}$ between rows and plants respectively. The experiment was laid out in Randomized Complete Block Design replicated thrice. All the recommended packages of practices were adopted to raise a good crop except irrigations as crop was irrigated through rains itself. The observations were recorded on five randomly selected plants from each of parents and $F_{1}$ crosses on fifteen yield and yield attributing traits. Heterosis over mid parent for yield and its components was calculated as usual procedure. Cross combinations namely; BAU-274-92×IR-36 gave significantly positive heterosis to the tune of $31.39 \%$ followed by BR- $8 \times$ IR-36 (27.57\%) and BR-8 $\times$ BD-202 (27\%) and BAU-211-90 $\times$ IR-36 (27.44\%), BAU-211 $\times$ BD$202(25.83 \%)$ and BAU-269-92 $\times$ IR-36 (25.97\%) for grain yield plant ${ }^{-1}$. The gca status of the parents involved revealed high $\times$ high, high $\times$ low and low $\times$ low combinations means involvement of both additive and non additive gene effects. The combinations can be further improved through simple selection procedures (additive $\times$ additive) or after advancing the generations through transgression effects.
\end{abstract}

\section{Introduction}

Rice is the basic staple for most of the people. More than $90 \%$ of rice is produced and consumed in Asia only. In the early 1960 's high yielding variety played a central role in the success of green revolution and enhance Asian rice production by more than triple in the past four plus decades from $200 \mathrm{mt}$ (paddy equivalent) in the early $1960 \mathrm{~s}$ to more than $600 \mathrm{mt}$ in 2010 . This has been possible with the introduction of modern varieties along with assured irrigation facility, subsidised inputs etc. According to the United Nations 2010 population projection there is addition of 700 billion people in next 30 years. 5 billion people will be added by 2035 and 5.15 billion by 2050 in Asia alone. On the basis of per capita consumption trends of Asia in last two decades, total consumption is expected to grow at the rate of population growth or may even exceeds as per the recent uptrend in three countries (China, India, and Indonesia) continue. The global consumption will probably rise from 439 $\mathrm{mt}$ (milled rice) in 2010 to $555 \mathrm{mt}$ in 2035 (FAO, 2012).

In the early period of green revolution (first three decades) witness higher productivity growth of more than $2 \%$ annually, that intended farmers to bring additional area into rice production. The current area under rice cultivation is all time high at around 160 mha compared with 120 mha in the early 1960 s. Further area expansion under rice cultivation cannot be possible for most of the rice growing countries. To meet the growing global needs, yield growth will have to be maintained at $1.2-1.5 \%$ to about 2020 and $1.0-1.2 \%$ annually beyond 2020 without any possibility of area expansion. Thus a combination of both productivity growth and increasing cropping intensity through adoption of high yielding short duration variety is needed. To achieve this goal exploitation of the heterosis or hybrid vigour could be the better option. Heterosis is the superiority of the $\mathrm{F}_{1} \mathrm{~s}$ over both the parents. Vanaja and Babu (2004) has also mentioned that yield increase in rice was due to favourable heterosis in flag leaf area, number of spikelets panicle $^{-1}$ and number of grains panicle ${ }^{-1}$. There is need to study various morphological traits to get better level of heterosis in different combination of geneotypes. Therefore, increasing its productivity is of high importance in breeding programs. 
Reduced plant height, moderate tillering, large and compact panicles, increased kernel number per panicle, increased thousand kernel weight and higher yield are the most important characters of rice that has to be improved in breeding programs (Mackill and Lei, 1997; Miller et al., 1993; Nemoto et al., 1995; Paterson et al., 2005; Wayne and Dilday, 2003). Since some rice hybrids show heterosis, it subsequently results to production in terms of yields which is 15 to $30 \%$ higher than inbred varieties (Yuan, 1994; Fujimura et al., 1996), and finding a better cross combination is of high importance. Most of the improved varieties hybrids utilize this phenomenon of hybrid vigour (Singh et al., 1999). Line $\times$ tester analysis is used to evaluate the general and specific combining ability of various lines and to estimate gene effects and it is useful in deciding the relative ability of female and male lines to produce desirable hybrid combinations (Kempthorne, 1957).

\section{Material and Methods}

The genetic material was comprised of fifteen diverse testers of rice as female crossed with three established lines as male. A total of 45 crosses were made during kharif 2011 (JuneDecember) at Crop Research station of Birsa agricultural University, Kanke, Ranchi. The method of analysis used was line $\times$ tester (Kampthorne, 1957). All the forty five $F_{1}$ hybrids along with eighteen parents were evaluated for heterosis over MP and BP in randomized block design in three replications in the next 2012 (June-December). The plot size was kept $5 \times 1$ $\mathrm{m}^{2}$ and spacing was $20 \times 15 \mathrm{~cm}^{2}$. Recommended agronomic practices were followed for raising good crop except irrigation pattern. The crop was grown solely on rain water even in extreme weather condition. Five randomly selected plants plot $^{-1}$ from parents and $\mathrm{F}_{1}$ plant population were tagged for recording observation on days to panicle emergence, plant height, total number of leaves plant ${ }^{-1}$, flag leaf area, total number of tillers plant $^{-1}$, total number of ear bearing tillers plant ${ }^{-1}$, panicle length, number of grains panicle ${ }^{-1}$, spikelet fertility (\%), grain yield plant ${ }^{-1}$, days to maturity and harvest index (\%). Relative heterosis and heterobeltiosis were calculated. Heterosis was estimated from mean values according to Fehr (1987) and t-test was performed.

\section{Results and Discussion}

The analysis of variance (Table $1 \mathrm{a} \& 1 \mathrm{~b}$ ) with sixty-three entries including parents and crosses in a line $\times$ tester design revealed significant varietal differences for all the fifteen quantitative characters. Both the parents as well as crosses were found to be significantly different for all the characters studied except panicle length. The differences among parents versus crosses were also highly significant for all the characters except the characters number of effective tillers plant ${ }^{-1}$ and panicle length. The similar results were also obtained by Reddy and De in 1996.

The general combining ability variance $\left(\sigma^{2}\right.$ gca) was reported significant for all the characters except for panicle length,

\begin{tabular}{lccccccccc}
\hline \multicolumn{2}{l}{ Table 1a: Analysis of variance of 15 quantitative characters in rice (m.s.s.) } \\
\hline $\begin{array}{l}\text { Sources of } \\
\text { variation }\end{array}$ & $\begin{array}{c}\text { D.F. } \\
\text { Days of panicle } \\
\text { emergence }\end{array}$ & $\begin{array}{c}\text { Plant } \\
\text { height }\end{array}$ & $\begin{array}{c}\text { Days to } \\
\text { maturity }\end{array}$ & $\begin{array}{c}\text { Total no. of } \\
\text { leaves plant }^{-1}\end{array}$ & $\begin{array}{c}\text { Flag } \\
\text { leaf area }\end{array}$ & $\begin{array}{c}\text { Total no. } \\
\text { tillers plant }{ }^{-1}\end{array}$ & $\begin{array}{c}\text { No. of effective } \\
\text { tillers plant }^{-1}\end{array}$ & $\begin{array}{c}\text { Panicle } \\
\text { length }^{\text {leng }}\end{array}$ \\
\hline Replication & 2 & 0.323 & 9.255 & 1.180 & 51.176 & 7.310 & 2.589 & 0.009 & 2.550 \\
Genotypes & 62 & $370.324^{* *}$ & $555.484^{* *}$ & $391.845^{* *}$ & $441.550^{* *}$ & $175.339^{* *}$ & $20.928^{* *}$ & $15.899^{* *}$ & $12.864^{*}$ \\
Parents & 17 & $456.646^{* *}$ & $671.628^{* *}$ & $447.882^{* *}$ & $355.326^{*}$ & $181.732^{* *}$ & $15.373^{*}$ & $7.790^{*}$ & 10.670 \\
Crosses & 44 & $391.085^{* *}$ & $506.830^{* *}$ & $355.328^{* *}$ & $459.239^{* *}$ & $136.409^{* *}$ & $22.231^{* *}$ & $19.043^{* *}$ & 13.812 \\
P vs C & 1 & $1157.57^{* *}$ & $721.812^{* *}$ & $1045.945^{* *}$ & $1129.067^{*}$ & $1779.582^{* *}$ & $58.039^{* *}$ & $15.435^{* *}$ & 8.453 \\
Error & 124 & 1.721 & 52.897 & 1.594 & 169.508 & 6.719 & 7.460 & 4.537 & 9.462 \\
CV \% & & 1.542 & 9.073 & 1.079 & 20.194 & 14.526 & 18.635 & 17.375 & 14.514 \\
\hline
\end{tabular}

\begin{tabular}{lcccccccc}
\hline \multicolumn{2}{l}{ Table 1b: Analysis of variance of 15 quantitative characters in rice (m.s.s.) } \\
\hline $\begin{array}{l}\text { Sources of } \\
\text { variation }\end{array}$ & $\begin{array}{c}\text { D.F. } \\
\begin{array}{c}\text { No. of grains } \\
\text { panicle }\end{array}\end{array}$ & $\begin{array}{c}\text { Spikelet } \\
\text { fertility (\%) }\end{array}$ & $\begin{array}{c}\text { Grain yield } \\
\text { panicle }^{-1}\end{array}$ & $\begin{array}{c}1000 \text { grain } \\
\text { weight }\end{array}$ & $\begin{array}{c}\text { Biological } \\
\text { yield }\end{array}$ & $\begin{array}{c}\text { Grain yield } \\
\text { plant }^{-1}\end{array}$ & $\begin{array}{c}\text { Harvest } \\
\text { index }\end{array}$ \\
\hline Replication & 2 & 170.110 & 25.857 & 0.016 & 0.043 & 7.429 & 1.762 & 3.300 \\
Genotypes & 62 & $1653.026^{* *}$ & $385.754^{* *}$ & $0.461^{* *}$ & $11.688^{* *}$ & $67.575^{* *}$ & $21.616^{* *}$ & $81.730^{* *}$ \\
Parents & 17 & $1484.923^{* *}$ & $116.656^{* *}$ & $0.425^{*}$ & $9.092^{* *}$ & $41.793^{* *}$ & $11.897^{* *}$ & $31.275^{* *}$ \\
Crosses & 44 & $1446.639^{* *}$ & $462.895^{* *}$ & $0.467^{* *}$ & $10.009^{* *}$ & $78.346^{* *}$ & $25.260^{* *}$ & $94.098^{* *}$ \\
P vs C & 1 & $10010.524^{* *}$ & $1566.219^{* *}$ & $0.826^{*}$ & $129.700^{* *}$ & $31.959^{*}$ & $26.522^{* *}$ & $395.301^{* *}$ \\
Error & 124 & 156.293 & 35.301 & 0.200 & 0.701 & 7.767 & 1.211 & 2.975 \\
CV \% & & 14.574 & 7.961 & 25.522 & 4.012 & 7.557 & 8.118 & 4.712 \\
\hline
\end{tabular}

"significant at $(p=0.05) ;{ }^{* *}$ significant at $(p=0.01)$ 
number of grains panicle ${ }^{-1}$, spikelet fertility, grain yield panicle $^{-1}, 1000$ grain weight and harvest index [Table 2a \& 2b]. The variances due to specific combining ability $\left(\sigma^{2} \mathrm{sca}\right)$ were also found significant for all the characters except the panicle length. The sca variance was higher than the corresponding gca variance for each character. The relative proportion $\sigma^{2}$ gca and $\sigma^{2}$ sca revealed that specific combining ability was higher for all the traits indicating the preponderance of non-additive gene action for these traits. These results are In general agreement with the findings of Saidaiah et al. (2010), Pratap et al. (2013) and Thakare et al. (2013).

The estimate of heterosis and heterobeltiosis of cross combinations for each character in desired direction are presented in table $3 \mathrm{a}, \mathrm{b}, \mathrm{c}, \mathrm{d} \&$ e. The crosses viz., BAU-274$92 \times$ IR-36, BR- $8 \times$ IR-36, BR- $8 \times$ BD -202 , BAU-211-90 $\times$ IR-36, BAU-211-90 $\times$ BD-202 and BAU-269-92 $\times$ IR-36 had shown desirable positive heterosis for grain yield as well as other yield related characters like biological yield, 1000 grains weight, total number of effective tillers plant ${ }^{-1}$, days of panicle emergence and days to maturity. These crosses also exhibited significant heterosis for grain yield panicle ${ }^{-1}$ except BAU-21190 $\times$ BD-202 and BAU-269-92 $\times$ IR-36 (Ali and Khan, 1995; Rao et al., 1996; Yolanda and Das, 1996; Singh and Haque, 1999; Vishwakarma et al., 1999). With regard to plant height, the three hybrids i.e., BR-8 BD-202, BAU-274-92×BD-202 and BAU198-90 $\times$ IR-64 had desirable relative negative heterosis. The crosses i.e. BAU-198-90 $\times$ IR- 64 and BR- $8 \times$ BD-202 had also shown desirable negative heterobeltiosis indicating the scope for breeding superior hybrids with desirable plant height. In the same way the crosses BAU-269-92×IR-36, and BAU-295$93 \times$ IR-36 had shown desirable significant relative heterosis for days to maturity and Mashuri $\times$ IR-36 and BAU-295-93×IR-36 exhibited significant heterobeltiosis showing further scope for developing early varieties. Similar results were obtained by scientists Ali and Khan (1995), Pandey et al. (1995), Rao et al. (1996), Yolanda and Das (1996), Singh and Haque (1999) and Vishwakarma et al. (1999). A considerable amount of relative heterosis was found only in BAU-205-90×BD-202, BAU211-90 $\times$ IR-36 and BR- $8 \times$ BD-202 for total number of leaves plant $^{-1}$ whereas single cross BAU-29-93 $\times$ IR-36 exhibited significant heterobeltiosis. The significant relative heterosis was observed maximum in BAU-294-93 $\times$ IR- 64 followed by IR-36-2 $\times$ IR-64 for flag leaf whereas IR-36-2 $\times$ IR- 64 had shown highest magnitude of heterosis over better parent. This is in accordance with other studies of Julfiquar and Tepora (1992), Xiong et al. (1996) and Singh and Haque (1999). Significant high heterosis was observed in BAU-211-90×BD-202 followed by BAU-211-90 $\times$ IR-36 over mid parent for the character total number of tillers plant ${ }^{-1}$. The crosses BAU-270-92 $\times$ BD-202 and BR- $8 \times$ BD-202 had shown desirable heterosis over better parent. This has also been reported by Ali and Khan (1995) and Reddy and Nerker (1995). The cross BAU-274-92×IR-36 had shown maximum heterosis over both mid and better parent for grain yield panicle ${ }^{-1}$ followed by Mahshuri $\times$ IR-36 whereas BAU-198-90×IR-36 exhibited only significant higher relative heterosis. Similar results were also observed in previous studies conducted by other workers (Ali and Khan, 1995; Pandey et al., 1995 and Quin et al., 1995). The desirable relative

\begin{tabular}{lcccccccc}
\hline \multicolumn{6}{l}{ Table 2a: Estimates of variance components and combining ability variances for all the 15 quantitative characters studied in rice } \\
\hline Characters & $\begin{array}{c}\text { Days of panicle } \\
\text { emergence }\end{array}$ & $\begin{array}{c}\text { Plant } \\
\text { height }\end{array}$ & $\begin{array}{c}\text { Days to } \\
\text { maturity }\end{array}$ & $\begin{array}{c}\text { Total no. of } \\
\text { leaves plant }{ }^{-1}\end{array}$ & $\begin{array}{c}\text { Flag } \\
\text { leaf area }\end{array}$ & $\begin{array}{c}\text { Total no. } \\
\text { tillers plant }{ }^{-1}\end{array}$ & $\begin{array}{c}\text { No. of effective } \\
\text { tillers plant }\end{array}$ & $\begin{array}{c}\text { Panicle } \\
\text { length }\end{array}$ \\
\hline$\sigma^{2} \mathrm{f}$ & $43.389^{* *}$ & $78.676^{* *}$ & $55.081^{* *}$ & $51.275^{*}$ & $16.463^{* *}$ & $2.545^{*}$ & $2.916^{* *}$ & 0.067 \\
$\sigma^{2} \mathrm{~m}$ & 4.365 & 2.135 & $4.233^{* *}$ & -6.674 & $15.645^{* *}$ & -0.265 & -0.111 & -0.122 \\
$\sigma^{2} \mathrm{fm}$ & $194.696^{* *}$ & $224.265^{* *}$ & $188.899^{* *}$ & $156.548^{* *}$ & $50.546^{* *}$ & $8.023^{* *}$ & $6.382^{* *}$ & 4.408 \\
$\sigma^{2} \mathrm{gca}$ & $1.375^{*}$ & $2.492^{* *}$ & $1.697^{* *}$ & $1.666^{* *}$ & $0.127^{*}$ & $0.082^{*}$ & $0.097^{*}$ & -0.010 \\
$\sigma^{2} \mathrm{sca}$ & $54.899^{* *}$ & $74.755^{* *}$ & $62.966^{* *}$ & $52.183^{* *}$ & $16.849^{* *}$ & $2.674^{* *}$ & $2.127^{* *}$ & 1.469 \\
$\sigma^{2} \mathrm{gca} / \sigma^{2} \mathrm{sca}$ & 0.021 & 0.033 & 0.027 & 0.032 & 0.008 & 0.031 & 0.045 & -0.007 \\
\hline
\end{tabular}

\begin{tabular}{lccccccc}
\hline \multicolumn{6}{l}{ Table 2b: Estimates of variance components and combining ability variances for all the 15 quantitative characters studied in rice } \\
\hline Characters & $\begin{array}{c}\text { No. of grains } \\
\text { panicle }{ }^{-1}\end{array}$ & $\begin{array}{c}\text { Spikelet fer- } \\
\text { tility (\%) }\end{array}$ & $\begin{array}{c}\text { Grain yield } \\
\text { panicle }\end{array}$ & $\begin{array}{c}1000 \text { grain } \\
\text { weight }\end{array}$ & $\begin{array}{c}\text { Biological } \\
\text { yield }\end{array}$ & $\begin{array}{c}\text { Grain yield } \\
\text { plant }^{-1}\end{array}$ & Harvest index \\
\hline$\sigma^{2} \mathrm{f}$ & -23.689 & -8.517 & $0.050^{*}$ & $0.952^{*}$ & $10.673^{* *}$ & $4.525^{* *}$ & $9.352^{*}$ \\
$\sigma^{2} \mathrm{~m}$ & 26.954 & 11.828 & 0.001 & 0.004 & -0.562 & -0.047 & -0.302 \\
$\sigma^{2} \mathrm{fm}$ & $1303.048^{* *}$ & $427.789^{*}$ & $0.122^{*}$ & $6.575^{* *}$ & $41.164^{* *}$ & $11.187^{* *}$ & $64.960^{* *}$ \\
$\sigma^{2} \mathrm{gca}$ & -3.255 & -1.155 & 0.001 & 0.026 & $0.347^{*}$ & $0.152^{*}$ & 0.270 \\
$\sigma^{2} \mathrm{sca}$ & $434.349^{*}$ & $142.596^{* *}$ & $0.041^{*}$ & $2.192^{* *}$ & $13.721^{* *}$ & $3.729^{* *}$ & $21.653^{* *}$ \\
$\sigma^{2} \mathrm{gca} / \sigma^{2} \mathrm{sca}$ & -0.007 & -0.008 & 0.035 & 0.012 & 0.025 & 0.041 & 0.012 \\
\hline
\end{tabular}

*significant at $(\mathrm{p}=0.05) ;{ }^{* *}$ significant at $(p=0.01)$ 
Table 3a: Estimates of heterosis (\%) over mid parent (mp) and better parent

\begin{tabular}{|c|c|c|c|c|c|c|}
\hline \multirow[t]{2}{*}{ Crosses } & \multicolumn{2}{|c|}{ Days of panicle emergence } & \multicolumn{2}{|c|}{ Plant height $(\mathrm{cm})$} & \multicolumn{2}{|c|}{ Days to maturity } \\
\hline & over mp & over bp & over mp & over bp & over mp & over bp \\
\hline BAU 198-90×IR-36 & $-12.580^{* *}$ & $-23.190^{* *}$ & 33.798 & 47.654 & $-6.165^{* *}$ & $-13.810^{* *}$ \\
\hline BAU 198-90×BD-202 & $-10.190^{* *}$ & $-20.590^{* *}$ & 5.527 & 10.284 & $-5.405^{* *}$ & $-13.220^{* *}$ \\
\hline BAU 198-90×IR-64 & $-5.979^{* *}$ & $-17.390^{* *}$ & $-21.093^{* *}$ & $-17.590^{* *}$ & $-11.410^{* *}$ & $-19.620^{* *}$ \\
\hline Mashuri×IR-36 & $-22.710^{* *}$ & $-30.430^{* *}$ & -9.837 & -0.423 & $-13.680^{* *}$ & $-20.920^{* *}$ \\
\hline Mashuri $\times$ BD-202 & $4.052^{* *}$ & $-6.960^{* *}$ & -5.998 & -1.840 & $7.018^{* *}$ & -1.840 \\
\hline Mashuri×IR-64 & 0.483 & $-9.570^{* *}$ & $-18.568^{*}$ & -14.892 & $7.311^{* *}$ & -0.460 \\
\hline BAU 270-92×IR-36 & 1.205 & $-8.700^{* *}$ & -3.642 & 4.495 & 0.148 & $-6.360^{* *}$ \\
\hline BAU 270-92×BD-202 & $-6.478^{* *}$ & $-15.080^{* *}$ & 6.304 & 34.893 & $-3.540^{* *}$ & $-9.920^{* *}$ \\
\hline BAU 270-92×IR-64 & $7.229^{* *}$ & $-3.260^{*}$ & 1.829 & 16.894 & $5.677^{* *}$ & -2.420 \\
\hline BAU 274-92×IR-36 & $8.350^{* *}$ & 1.090 & 6.154 & 21.192 & $6.017^{* *}$ & 2.210 \\
\hline BAU 274-92×BD-202 & $-7.241^{* *}$ & $-12.870^{* *}$ & -21.437 & 5.604 & $-8.441^{* *}$ & $-11.850^{* *}$ \\
\hline BAU 274-92×IR-64 & $-16.12^{* *}$ & $-21.740^{* *}$ & -13.729 & 4.506 & $-13.560^{* *}$ & $-17.740^{* *}$ \\
\hline BAU 291-93×IR-36 & $7.634^{* *}$ & 2.170 & 11.450 & 25.998 & 1.420 & -1.380 \\
\hline BAU 291-93×BD-202 & $-13.080^{* *}$ & $-16.910^{* *}$ & -10.101 & 19.525 & $-4.397^{* *}$ & $-7.160^{* *}$ \\
\hline BAU 291-93×IR-64 & $-11.070^{* *}$ & $-15.580^{* *}$ & 41.700 & 69.903 & $-6.723^{* *}$ & $-10.480^{* *}$ \\
\hline BAU 294-93×IR-36 & $-7.353^{* *}$ & $-8.700^{* *}$ & -12.083 & 1.459 & $-4.709^{* *}$ & $-4.980^{* *}$ \\
\hline BAU $294-93 \times$ BD-202 & $-7.778^{* *}$ & $-8.460^{* *}$ & -12.357 & -12.071 & $-6.224^{* *}$ & $-6.610^{* *}$ \\
\hline BAU 294-93×IR-64 & $-20.590^{* *}$ & $-21.740^{* *}$ & -11.292 & -3.318 & $-16.940^{* *}$ & $-18.280^{* *}$ \\
\hline BAU 295-93×IR-36 & $-25.930^{* *}$ & $-27.840^{* *}$ & -0.230 & 7.864 & $-17.430^{* *}$ & $-19.790^{* *}$ \\
\hline BAU 295-93×BD-202 & $-17.940^{* *}$ & $-20.620^{* *}$ & -5.975 & 18.898 & $-11.110^{* *}$ & $-13.540^{* *}$ \\
\hline BAU 295-93×IR-64 & $-22.750^{* *}$ & $-24.740^{* *}$ & -1.738 & 12.437 & $-13.760^{* *}$ & $-15.100^{* *}$ \\
\hline BAU 213-92×IR-36 & $-21.550^{* *}$ & $-23.450^{* *}$ & -12.827 & -11.046 & $-14.320^{* *}$ & $-16.140^{* *}$ \\
\hline BAU 213-92×BD-202 & $14.230^{* *}$ & $10.690^{* *}$ & $-17.399^{*}$ & -2.141 & $11.470^{* *}$ & $9.260^{* *}$ \\
\hline BAU 213-92×IR-64 & $6.007^{* *}$ & $3.440^{*}$ & $-15.833^{*}$ & -9.354 & $8.000^{* *}$ & $7.140^{* *}$ \\
\hline BAU $200-90 \times$ IR-36 & $-13.860^{* *}$ & $-20.910^{* *}$ & 8.872 & 31.493 & $-11.420^{* *}$ & $-18.850^{* *}$ \\
\hline BAU $200-90 \times$ BD-202 & $-11.300^{* *}$ & $-19.090^{* *}$ & -1.777 & 40.574 & $-5.013^{* *}$ & $-12.870^{* *}$ \\
\hline BAU 200-90×IR-64 & $-13.860^{* *}$ & $-20.910^{* *}$ & -1.508 & 26.529 & $-8.798^{* *}$ & $-15.400^{* *}$ \\
\hline BAU 205-90×IR-36 & 1.840 & $-9.780^{* *}$ & 6.581 & 6.677 & $3.428^{* *}$ & $-4.150^{* *}$ \\
\hline BAU $205-90 \times$ BD-202 & $15.050^{* *}$ & 2.570 & -12.145 & 1.631 & $8.929^{* *}$ & 0.830 \\
\hline BAU 205-90×IR-64 & $11.660^{* *}$ & -1.090 & -7.256 & -2.297 & $10.430^{* *}$ & 1.080 \\
\hline BR- $8 \times$ IR-36 & $-20.210^{* *}$ & $-21.880^{* *}$ & $-18.227^{*}$ & -14.795 & $-8.540^{* *}$ & $-8.790^{* *}$ \\
\hline BR-8×BD-202 & $-18.570^{* *}$ & $-20.830^{* *}$ & $-24.848^{* *}$ & $-16.805^{*}$ & $-5.365^{* *}$ & $-5.490^{* *}$ \\
\hline BR-8×IR-64 & $-22.340^{* *}$ & $-23.960^{* *}$ & $-18.455^{*}$ & $-17.515^{*}$ & $-8.967^{* *}$ & $-9.950^{* *}$ \\
\hline IR-36-2×IR-36 & -1.124 & $-4.350^{* *}$ & 1.707 & 23.101 & -2.145 & $-4.950^{* *}$ \\
\hline IR-36-2×BD-202 & $3.019^{*}$ & 0.360 & 4.786 & 50.322 & 0.669 & -2.080 \\
\hline IR-36-2×IR-64 & $-6.742^{* *}$ & $-9.780^{* *}$ & 36.574 & 75.842 & $-6.085^{* *}$ & $-7.550^{* *}$ \\
\hline BAU 211-90×IR-36 & $19.050^{* *}$ & $8.700^{* *}$ & -4.972 & -3.938 & $9.091^{* *}$ & $4.420^{* *}$ \\
\hline BAU 211-90×BD-202 & $20.000^{* *}$ & $10.290^{* *}$ & -1.119 & 15.904 & $10.090^{* *}$ & $5.230^{* *}$ \\
\hline BAU 211-90×IR-64 & 1.190 & $-7.610^{* *}$ & -4.426 & 1.919 & -0.996 & $-6.450^{* *}$ \\
\hline BAU 269-92×IR-36 & $-23.400^{* *}$ & $-25.000^{* *}$ & 38.708 & 49.310 & $-18.160^{* *}$ & $-19.680^{* *}$ \\
\hline BAU 269-92×BD-202 & $-20.710^{* *}$ & $-22.920^{* *}$ & 22.727 & 54.438 & $-10.960^{* *}$ & $-12.500^{* *}$ \\
\hline BAU 269-92×IR-64 & $-23.400^{* *}$ & $-25.000^{* *}$ & 27.792 & 45.562 & $-16.580^{* *}$ & $-17.020^{* *}$ \\
\hline BAU 275-92×IR-36 & $-7.778^{* *}$ & $-9.780^{* *}$ & 17.545 & 21.220 & $-12.930^{* *}$ & $-14.210^{* *}$ \\
\hline BAU 275-92×BD-202 & $-4.851^{* *}$ & $-6.250^{* *}$ & 9.353 & 22.383 & $-5.163^{* *}$ & $-6.430^{* *}$ \\
\hline
\end{tabular}


International Journal of Bio-resource and Stress Management 2015, 6(1):051-062

\begin{tabular}{lcccccc}
\hline & \multicolumn{7}{c}{} & & & \\
\hline BAU $275-92 \times$ IR-64 & $-11.110^{* *}$ & $-13.040^{* *}$ & 14.184 & 16.667 & $-15.170^{* *}$ & $-15.280^{* *}$ \\
$\mathrm{SEm} \pm$ & 0.930 & 1.070 & 5.140 & 5.940 & 0.890 & 1.030 \\
$\mathrm{CD}(p=0.05)$ & 2.570 & 2.970 & 14.250 & 16.460 & 2.470 & 2.860 \\
$\mathrm{CD}(p=0.01)$ & 3.380 & 3.900 & 18.730 & 21.630 & 3.250 & 3.750 \\
\hline
\end{tabular}

*significant at $(p=0.05) ;{ }^{* *}$ significant at $(p=0.01)$

\begin{tabular}{|c|c|c|c|c|c|c|}
\hline \multirow[t]{2}{*}{ Crosses } & \multicolumn{2}{|c|}{ Total number of leaves plant ${ }^{-1}$} & \multicolumn{2}{|c|}{ Flag leaf area } & \multicolumn{2}{|c|}{ Total number of tillers plant ${ }^{-1}$} \\
\hline & over mp & over bp & over mp & over bp & over mp & over bp \\
\hline BAU 198-90×IR-36 & -14.561 & -17.240 & -63.540 & -71.124 & -14.286 & -16.092 \\
\hline BAU 198-90×BD-202 & 8.840 & -0.086 & -70.385 & -71.901 & $10.675^{* *}$ & 1.580 \\
\hline BAU 198-90×IR-64 & -23.344 & -29.472 & -45.444 & -55.140 & -22.284 & -27.974 \\
\hline Mashuri×IR-36 & 6.673 & -4.920 & -80.593 & -81.068 & $10.448^{* *}$ & -0.766 \\
\hline Mashuri×BD-202 & 6.131 & 6.085 & -63.459 & -69.222 & $7.434^{* *}$ & $7.203^{*}$ \\
\hline Mashuri×IR-64 & -13.787 & -26.654 & -21.746 & -23.639 & -12.974 & -25.623 \\
\hline BAU 270-92×IR-36 & -11.776 & -18.515 & -24.742 & -37.459 & -8.299 & -15.326 \\
\hline BAU 270-92×BD-202 & 16.832 & -14.620 & -81.086 & -81.244 & $17.209^{* *}$ & $14.053^{* *}$ \\
\hline BAU 270-92×IR-64 & -2.165 & -3.985 & -39.325 & -47.496 & 2.724 & -9.882 \\
\hline BAU 274-92×IR-36 & 2.734 & 5.527 & -18.859 & -26.691 & 5.055 & -8.429 \\
\hline BAU 274-92×BD-202 & -32.181 & -53.692 & -68.828 & -71.844 & -32.506 & -34.913 \\
\hline BAU 274-92×IR-64 & -13.462 & -19.704 & -15.729 & -20.339 & -12.936 & -27.633 \\
\hline BAU 291-93×IR-36 & 7.965 & $32.335^{*}$ & -14.314 & -30.413 & $8.119^{* *}$ & 4.598 \\
\hline BAU 291-93×BD-202 & -36.259 & 51.331 & -36.362 & -37.651 & -37.307 & -41.779 \\
\hline BAU 291-93×IR-64 & -19.282 & -17.835 & $13.494^{* *}$ & -4.157 & -10.987 & -18.416 \\
\hline BAU 294-93×IR-36 & -34.541 & -33.241 & -40.758 & -57.804 & -34.008 & -37.548 \\
\hline BAU 294-93×BD-202 & -32.753 & -49.960 & -10.978 & -43.189 & -27.149 & -30.886 \\
\hline BAU 294-93×IR-64 & -31.940 & -32.195 & $80.065^{* *}$ & $24.567^{* *}$ & -30.418 & -37.532 \\
\hline BAU 295-93×IR-36 & -48.434 & -41.764 & -19.104 & -28.159 & -47.490 & -47.893 \\
\hline BAU $295-93 \times$ BD-202 & -20.231 & -36.936 & -20.419 & -40.111 & -19.313 & -26.834 \\
\hline BAU 295-93×IR-64 & -27.199 & -23.698 & $35.338^{* *}$ & $15.167^{* *}$ & -26.182 & -30.705 \\
\hline BAU 213-92×IR-36 & -16.126 & -28.333 & -77.264 & -78.945 & -16.592 & -28.736 \\
\hline BAU 213-92×BD-202 & 1.456 & -33.661 & -56.401 & -66.111 & $6.091^{*}$ & 0.024 \\
\hline BAU 213-92×IR-64 & -13.662 & -22.678 & -14.311 & -24.134 & -13.389 & -29.339 \\
\hline BAU 200-90×IR-36 & -34.249 & -19.514 & -45.348 & -46.089 & -29.762 & -32.184 \\
\hline BAU $200-90 \times$ BD-202 & -12.088 & -32.975 & -36.985 & -48.544 & -13.717 & -19.753 \\
\hline BAU 200-90×IR-64 & -33.222 & -32.110 & $10.230^{* *}$ & 3.618 & -31.343 & -37.191 \\
\hline BAU 205-90×IR-36 & -7.633 & 0.278 & $23.388^{* *}$ & 18.289 & -3.178 & -5.440 \\
\hline BAU 205-90×BD-202 & $32.536^{*}$ & 5.488 & -60.008 & -68.067 & $14.286^{* *}$ & 0.767 \\
\hline BAU 205-90×IR-64 & -12.938 & -8.699 & -21.673 & -28.340 & -8.642 & -11.589 \\
\hline BR- $8 \times$ IR-36 & $8.092^{*}$ & -5.643 & -44.784 & -54.210 & 1.856 & -5.364 \\
\hline BR- $8 \times$ BD-202 & 28.139 & -12.944 & -34.093 & -34.467 & $17.321^{* *}$ & $13.418^{* *}$ \\
\hline BR-8×IR-64 & 1.241 & -6.449 & -3.190 & -16.414 & -2.901 & -14.320 \\
\hline IR-36-2×IR-36 & -24.989 & -10.294 & $11.745^{* *}$ & 0.000 & -23.517 & -28.352 \\
\hline IR-36-2×BD-202 & 5.333 & -21.779 & 4.712 & -20.733 & $6.178^{*}$ & 1.754 \\
\hline IR-36-2×IR-64 & -4.656 & -5.175 & $63.456^{* *}$ & $40.105^{* *}$ & -4.415 & -15.003 \\
\hline BAU 211-90×IR-36 & $35.481^{* *}$ & 21.504 & -27.225 & -29.064 & $22.467^{* *}$ & $6.513^{*}$ \\
\hline BAU 211-90×BD-202 & -12.106 & -12.106 & -64.013 & -70.889 & $26.368^{* *}$ & $11.404^{* *}$ \\
\hline BAU 211-90×IR-64 & -29.917 & -35.509 & -42.311 & -46.382 & -30.041 & -41.970 \\
\hline
\end{tabular}




\begin{tabular}{|c|c|c|c|c|c|c|}
\hline BAU 269-92×IR-36 & -8.156 & 23.983 & -45.180 & -45.423 & -2.174 & -7.216 \\
\hline BAU 269-92×BD-202 & -23.053 & -34.537 & -60.473 & -67.489 & -21.600 & -32.646 \\
\hline BAU 269-92×IR-64 & -24.648 & -24.613 & -37.409 & -40.646 & -23.288 & -23.536 \\
\hline BAU 275-92×IR-36 & -24.987 & -44.051 & -53.414 & -64.942 & -26.622 & -37.165 \\
\hline BAU 275-92×BD-202 & -4.935 & -38.383 & -44.809 & -50.983 & -9.367 & -14.334 \\
\hline BAU 275-92×IR-64 & -35.213 & -42.391 & -5.553 & -26.453 & -36.117 & -47.773 \\
\hline $\operatorname{SEm} \pm$ & 9.210 & 10.630 & 1.830 & 2.120 & 1.930 & 2.230 \\
\hline $\mathrm{CD}(p=0.05)$ & 25.510 & 29.460 & 5.080 & 5.870 & 5.350 & 6.180 \\
\hline $\mathrm{CD}(p=0.01)$ & 33.530 & 38.720 & 6.680 & 7.710 & 7.030 & 8.120 \\
\hline
\end{tabular}

*significant at $(p=0.05) ;{ }^{* *}$ significant at $(p=0.01)$

\begin{tabular}{|c|c|c|c|c|c|c|}
\hline \multirow[t]{2}{*}{ Crosses } & \multicolumn{2}{|c|}{ Total number of effective tillers plant ${ }^{-1}$} & \multicolumn{2}{|c|}{ Panicle length } & \multicolumn{2}{|c|}{ No. of grains panicle ${ }^{-1}$} \\
\hline & over mp & over bp & over $\mathrm{mp}$ & over bp & over mp & over bp \\
\hline BAU 198-90×IR-36 & -11.164 & -13.426 & $24.292^{* *}$ & $16.807^{* *}$ & -6.624 & -24.083 \\
\hline BAU 198-90×BD-202 & $11.227^{* *}$ & 3.877 & 3.874 & 1.401 & -21.240 & -29.942 \\
\hline BAU 198-90×IR-64 & -26.484 & -31.020 & -7.765 & -18.487 & -23.045 & -37.052 \\
\hline Mashuri×IR-36 & $10.026^{* *}$ & -0.926 & -16.618 & -26.098 & -22.681 & -24.456 \\
\hline Mashuri×BD-202 & $9.972^{* *}$ & $6.336^{* *}$ & -6.180 & -13.695 & -25.128 & -30.837 \\
\hline Mashuri×IR-64 & -2.956 & -15.596 & -14.551 & -28.682 & -3.365 & -5.118 \\
\hline BAU 270-92×IR-36 & -7.463 & -13.889 & 0.761 & -3.470 & -0.260 & -14.809 \\
\hline BAU 270-92×BD-202 & $21.978^{* *}$ & 19.355 & -7.467 & -7.845 & -28.559 & -19.433 \\
\hline BAU 270-92×IR-64 & $9.308^{* *}$ & -1.885 & -3.404 & -13.094 & -9.249 & -13.463 \\
\hline BAU 274-92×IR-36 & $7.013^{* *}$ & -4.630 & -0.625 & -2.304 & -7.562 & -17.970 \\
\hline BAU 274-92×BD-202 & -36.599 & -39.394 & -4.805 & -6.737 & -88.017 & -86.909 \\
\hline BAU 274-92×IR-64 & -14.428 & -26.307 & $8.333^{* *}$ & -0.154 & -35.340 & -38.596 \\
\hline BAU 291-93×IR-36 & $9.880^{* *}$ & $5.556^{*}$ & -3.135 & -4.630 & -29.270 & -38.214 \\
\hline BAU 291-93×BD-202 & -32.626 & -36.197 & -6.024 & -8.208 & -71.812 & -70.889 \\
\hline BAU 291-93×IR-64 & -5.556 & -12.596 & $32.776^{* *}$ & $22.531^{* *}$ & $50.205^{* *}$ & $64.017^{* *}$ \\
\hline BAU 294-93×IR-36 & -36.232 & -38.889 & 2.229 & 0.611 & -15.560 & -36.495 \\
\hline BAU 294-93×BD-202 & -34.043 & -37.374 & $6.116^{*}$ & 2.089 & -18.487 & -15.312 \\
\hline BAU 294-93×IR-64 & -33.643 & -38.732 & $14.286^{* *}$ & 5.313 & 0.037 & -5.843 \\
\hline BAU 295-93×IR-36 & -45.199 & -45.833 & -2.857 & -7.815 & -14.880 & -34.165 \\
\hline BAU 295-93×BD-202 & -25.450 & -31.296 & -1.449 & -9.974 & -14.504 & -8.943 \\
\hline BAU 295-93×IR-64 & -39.640 & -42.588 & 2.342 & -10.944 & -26.316 & -28.694 \\
\hline BAU 213-92×IR-36 & -11.172 & -24.537 & $38.538^{* *}$ & $33.014^{* *}$ & 2.009 & -31.004 \\
\hline BAU 213-92×BD-202 & $4.559^{*}$ & -5.234 & -7.006 & -14.092 & -4.510 & -9.621 \\
\hline BAU 213-92×IR-64 & -8.333 & -24.593 & $8.897^{* *}$ & -4.045 & -2.031 & -16.994 \\
\hline BAU 200-90×IR-36 & -20.000 & -25.000 & 2.694 & -2.711 & -9.155 & -35.330 \\
\hline BAU $200-90 \times$ BD-202 & -12.262 & -14.815 & 4.194 & -4.942 & -14.305 & -15.176 \\
\hline BAU 200-90×IR-64 & -32.701 & -39.160 & $6.137^{*}$ & -7.808 & -0.077 & -10.937 \\
\hline BAU 205-90×IR-36 & -2.059 & -3.146 & $7.143^{*}$ & 5.263 & 15.058 & -12.701 \\
\hline BAU $205-90 \times$ BD-202 & $15.789^{* *}$ & $4.549^{* *}$ & -3.738 & -9.091 & -39.566 & -36.721 \\
\hline BAU 205-90×IR-64 & -3.524 & -6.170 & $6.944^{* *}$ & -3.418 & -8.373 & -23.720 \\
\hline BR- $8 \times$ IR-36 & $6.203^{* *}$ & -0.926 & -9.360 & -11.694 & -11.190 & -23.405 \\
\hline BR-8×BD-202 & $28.219^{* *}$ & $25.100^{* *}$ & -7.402 & -13.504 & -22.713 & -19.194 \\
\hline BR-8×IR-64 & $5.238^{*}$ & -5.313 & $8.260^{* *}$ & -3.418 & -17.332 & -10.868 \\
\hline IR-36-2×IR-36 & -15.869 & -22.685 & 3.183 & -1.754 & -7.066 & -20.854 \\
\hline
\end{tabular}


International Journal of Bio-resource and Stress Management 2015, 6(1):051-062

\begin{tabular}{|c|c|c|c|c|c|c|}
\hline IR-36-2×BD-202 & $14.763^{* *}$ & $13.499^{* *}$ & -1.445 & -9.679 & -32.166 & -21.274 \\
\hline IR-36-2×IR-64 & $5.797^{* *}$ & -6.170 & $20.646^{* *}$ & 5.632 & -55.074 & -52.165 \\
\hline BAU $211-90 \times$ IR-36 & $29.947^{* *}$ & $12.500^{* *}$ & 2.640 & -0.797 & -39.712 & -39.712 \\
\hline BAU 211-90×BD-202 & $27.381^{* *}$ & $17.906^{* *}$ & 0.316 & -6.737 & -29.472 & -29.472 \\
\hline BAU 211-90×IR-64 & -25.831 & -37.875 & $11.307^{* *}$ & -1.223 & -18.372 & -30.277 \\
\hline BAU 269-92×IR-36 & $7.551^{* *}$ & $6.359^{* *}$ & $12.539^{* *}$ & $10.802^{* *}$ & -23.889 & -44.426 \\
\hline BAU 269-92×BD-202 & -16.792 & -24.870 & $10.241^{* *}$ & $7.679^{*}$ & -40.161 & -39.431 \\
\hline BAU 269-92×IR-64 & -21.145 & -23.308 & 2.341 & -5.556 & -12.801 & -20.297 \\
\hline BAU 275-92×IR-36 & -22.581 & -33.333 & -8.006 & -9.274 & -20.898 & -42.318 \\
\hline BAU 275-92×BD-202 & -7.784 & -15.152 & 1.357 & -1.147 & -39.286 & -38.618 \\
\hline BAU 275-92×IR-64 & -34.190 & -45.159 & 3.183 & -4.629 & -11.857 & -35.164 \\
\hline $\operatorname{SEm} \pm$ & 1.510 & 1.740 & 2.180 & 2.510 & 8.840 & 10.210 \\
\hline $\mathrm{CD}(p=0.05)$ & 4.170 & 4.820 & 6.030 & 6.960 & 24.500 & 28.290 \\
\hline $\mathrm{CD}(p=0.01)$ & 5.490 & 6.330 & 7.920 & 9.150 & 32.200 & 37.180 \\
\hline
\end{tabular}

* significant at $(p=0.05) ;{ }^{* *}$ significant at $(p=0.01)$

\begin{tabular}{|c|c|c|c|c|c|c|}
\hline \multirow[t]{2}{*}{ Crosses } & \multicolumn{2}{|c|}{ Spikelet fertility (\%) } & \multicolumn{2}{|c|}{ Grain yield panicle ${ }^{-1}$} & \multicolumn{2}{|c|}{1000 grain weight } \\
\hline & over $\mathrm{mp}$ & over bp & over $\mathrm{mp}$ & over bp & over $\mathrm{mp}$ & over bp \\
\hline BAU 198-90×IR-36 & 0.967 & -3.605 & $47.019^{* *}$ & $20.920^{* *}$ & $14.555^{* *}$ & $12.463^{* *}$ \\
\hline BAU 198-90×BD-202 & -3.548 & -8.738 & $14.695^{* *}$ & $2.910^{* *}$ & $4.923^{* *}$ & $0.462^{* *}$ \\
\hline BAU 198-90×IR-64 & -1.015 & -3.745 & $3.823^{* *}$ & -1.753 & $11.142^{* *}$ & $3.912^{* *}$ \\
\hline Mashuri×IR-36 & -1.799 & -2.142 & 58.799 & $45.865^{* *}$ & $12.456^{* *}$ & $10.029^{* *}$ \\
\hline Mashuri×BD-202 & -0.394 & -0.984 & $40.678^{* *}$ & $17.374^{* *}$ & $13.324^{* *}$ & $8.148^{* *}$ \\
\hline Mashuri×IR-64 & -4.884 & -6.986 & $25.366^{* *}$ & 0.180 & $3.042^{* *}$ & -3.971 \\
\hline BAU 270-92×IR-36 & -2.319 & -6.091 & -29.686 & -40.684 & $11.397^{* *}$ & $10.159^{* *}$ \\
\hline BAU 270-92×BD-202 & -8.143 & -12.485 & $35.185^{* *}$ & $24.786^{* *}$ & $12.875^{* *}$ & $8.842^{* *}$ \\
\hline BAU 270-92×IR-64 & -1.634 & -3.665 & $12.358^{* *}$ & $9.573^{* *}$ & -9.588 & -14.886 \\
\hline BAU 274-92×IR-36 & 1.575 & 0.190 & $64.507^{* *}$ & $46.366^{* *}$ & $15.212^{* *}$ & $9.266^{* *}$ \\
\hline BAU 274-92×BD-202 & -78.650 & -79.136 & -34.745 & -47.071 & -9.775 & -16.466 \\
\hline BAU 274-92×IR-64 & -13.727 & -14.176 & $36.806^{* *}$ & $6.486^{* *}$ & $13.853^{* *}$ & $3.018^{* *}$ \\
\hline BAU 291-93×IR-36 & -35.923 & -19.026 & $28.585^{* *}$ & $3.994^{* *}$ & $11.637^{* *}$ & $6.329^{* *}$ \\
\hline BAU 291-93×BD-202 & -56.982 & -58.943 & -34.729 & -42.550 & -4.283 & -11.014 \\
\hline BAU 291-93×IR-64 & 10.366 & 8.282 & -1.408 & -8.602 & $3.298^{* *}$ & -6.154 \\
\hline BAU 294-93×IR-36 & -2.476 & -2.862 & -23.391 & -34.896 & -9.898 & -10.110 \\
\hline BAU 294-93×BD-202 & -0.188 & -0.738 & -30.970 & -35.764 & -6.193 & -8.765 \\
\hline BAU 294-93×IR-64 & -13.434 & -15.386 & -28.685 & -29.861 & $0.232^{* *}$ & -4.850 \\
\hline BAU 295-93×IR-36 & 2.837 & -5.615 & -24.095 & -35.273 & $2.999^{* *}$ & -1.347 \\
\hline BAU $295-93 \times$ BD-202 & 2.099 & -5.493 & -24.906 & -29.806 & 6.056 & -0.863 \\
\hline BAU 295-93×IR-64 & -22.411 & -13.002 & -31.668 & -32.451 & -5.029 & -13.260 \\
\hline BAU 213-92×IR-36 & -10.805 & -12.961 & -5.137 & -15.400 & -2.647 & -5.096 \\
\hline BAU 213-92×BD-202 & -17.510 & -18.767 & -12.103 & -13.645 & 1.288 & -3.681 \\
\hline BAU 213-92×IR-64 & -10.393 & -14.156 & -7.016 & -10.450 & -8.937 & -15.429 \\
\hline BAU 200-90×IR-36 & -11.095 & -15.947 & -17.093 & -29.688 & $7.861^{* *}$ & $5.597^{* *}$ \\
\hline BAU $200-90 \times$ BD-202 & 5.197 & 0.335 & -17.757 & -23.611 & $10.637^{* *}$ & $10.151^{* *}$ \\
\hline BAU 200-90×IR-64 & -2.293 & -9.256 & -1.326 & -3.125 & $4.066^{*}$ & 1.070 \\
\hline BAU 205-90×IR-36 & 7.987 & 2.487 & $3.339^{* *}$ & -20.325 & -0.830 & -2.061 \\
\hline BAU 205-90×BD-202 & -6.640 & -10.608 & $3.981^{* *}$ & -13.279 & -11.948 & -15.203 \\
\hline
\end{tabular}




\begin{tabular}{|c|c|c|c|c|c|c|}
\hline BAU 205-90×IR-64 & -0.440 & -7.186 & -13.777 & -24.526 & -9.038 & -14.476 \\
\hline BR- $8 \times$ IR-36 & -5.285 & -8.138 & $6.572^{* *}$ & -10.374 & $3.866^{* *}$ & $3.774^{* *}$ \\
\hline BR- $8 \times$ BD-202 & -1.013 & -4.866 & $2.957^{* *}$ & -5.272 & $7.182^{* *}$ & $4.590^{* *}$ \\
\hline BR-8×IR-64 & -8.807 & -9.885 & 3.237 & 0.340 & -13.277 & -17.407 \\
\hline IR-36-2×IR-36 & -6.672 & -9.059 & -9.323 & -25.243 & $18.710^{* *}$ & $14.963^{* *}$ \\
\hline IR-36-2×BD-202 & -12.576 & -15.588 & $5.216^{* *}$ & -5.340 & $20.427^{* *}$ & $13.786^{* *}$ \\
\hline IR-36-2×IR-64 & -30.847 & -31.342 & $6.053^{* *}$ & 0.647 & -5.160 & -12.469 \\
\hline BAU 211-90×IR-36 & 3.430 & -1.843 & $33.954^{* *}$ & $14.109^{* *}$ & $16.866^{* *}$ & $11.214^{* *}$ \\
\hline BAU $211-90 \times$ BD-202 & -5.099 & -9.138 & -18.980 & -24.339 & $18.614^{* *}$ & $10.182^{* *}$ \\
\hline BAU 211-90×IR-64 & -11.005 & -17.139 & $25.893^{* *}$ & $24.339^{* *}$ & 1.445 & -7.912 \\
\hline BAU 269-92×IR-36 & -16.630 & -17.133 & -4.981 & -22.741 & $13.896^{* *}$ & $3.083^{* *}$ \\
\hline BAU $269-92 \times$ BD-202 & -12.042 & -6.427 & -16.095 & -25.701 & $2.081^{*}$ & -9.689 \\
\hline BAU 269-92×IR-64 & -6.140 & -8.443 & -28.214 & -33.022 & -5.458 & -18.154 \\
\hline BAU 275-92×IR-36 & -3.890 & -8.589 & -25.624 & -40.969 & $21.204^{* *}$ & $5.891^{* *}$ \\
\hline BAU $275-92 \times$ BD-202 & -15.180 & -18.610 & -36.627 & -45.374 & $28.000^{* *}$ & $9.427^{* *}$ \\
\hline BAU 275-92×IR-64 & -1.965 & -8.415 & -38.138 & -43.906 & $20.262^{* *}$ & 0.703 \\
\hline $\operatorname{SEm} \pm$ & 4.200 & 4.850 & 0.320 & 0.360 & 0.590 & 0.680 \\
\hline $\mathrm{CD}(p=0.05)$ & 11.640 & 13.440 & 0.880 & 1.010 & 1.640 & 1.890 \\
\hline $\mathrm{CD}(p=0.01)$ & 15.300 & 17.670 & 1.150 & 1.330 & 2.160 & 2.490 \\
\hline
\end{tabular}

*significant at $(p=0.05) ;{ }^{* *}$ significant at $(p=0.01)$

\begin{tabular}{|c|c|c|c|c|c|c|}
\hline \multirow[t]{2}{*}{ Crosses } & \multicolumn{2}{|c|}{ Biological yield } & \multicolumn{2}{|c|}{ Grain yield plant $^{-1}$} & \multicolumn{2}{|c|}{ Harvest index } \\
\hline & over $\mathrm{mp}$ & over bp & over $\mathrm{mp}$ & over bp & over $\mathrm{mp}$ & over bp \\
\hline BAU 198-90×IR-36 & $19.708^{* *}$ & $11.486^{* *}$ & $17.066^{* *}$ & $7.368^{* *}$ & -1.893 & -7.543 \\
\hline BAU 198-90×BD-202 & $15.680^{* *}$ & 6.256 & $7.052^{* *}$ & -0.199 & -7.166 & -10.053 \\
\hline BAU 198-90×IR-64 & -9.852 & -13.104 & -17.876 & -16.473 & -9.104 & -10.983 \\
\hline Mashuri×IR-36 & $7.853^{* *}$ & -1.590 & $11.312^{* *}$ & $7.088^{* *}$ & 2.412 & -5.396 \\
\hline Mashuri $\times$ BD-202 & -4.728 & -14.235 & $2.030^{*}$ & 0.000 & $6.090^{* *}$ & -4.531 \\
\hline Mashuri×IR-64 & -18.857 & -20.053 & -24.152 & -30.669 & -6.586 & -19.704 \\
\hline BAU $270-92 \times$ IR-36 & $12.883^{* *}$ & $11.158^{* *}$ & $12.100^{* *}$ & $3.842^{* *}$ & -0.468 & -7.215 \\
\hline BAU 270-92×BD-202 & $24.906^{* *}$ & $21.204^{* *}$ & $25.354^{* *}$ & $18.131^{* *}$ & 0.719 & -3.500 \\
\hline BAU 270-92×IR-64 & $24.083^{* *}$ & $13.263^{* *}$ & $16.507^{* *}$ & $13.687^{* *}$ & -5.960 & -6.826 \\
\hline BAU 274-92×IR-36 & $16.005^{* *}$ & $8.018^{*}$ & $31.387^{* *}$ & $35.166^{* *}$ & $12.665^{* *}$ & $6.096^{* *}$ \\
\hline BAU 274-92×BD-202 & $34.277^{* *}$ & $23.317^{* *}$ & -48.285 & -42.798 & -61.618 & -64.811 \\
\hline BAU 274-92×IR-64 & -14.036 & -17.130 & -15.596 & -22.900 & -1.680 & -13.990 \\
\hline BAU 291-93×IR-36 & $50.564^{* *}$ & $39.850^{* *}$ & $24.654^{* *}$ & $12.674^{* *}$ & -25.803 & -28.664 \\
\hline BAU 291-93×BD-202 & 1.026 & -7.447 & -36.631 & -41.761 & -37.062 & -37.749 \\
\hline BAU 291-93×IR-64 & -5.989 & -9.137 & -9.533 & -9.294 & -4.614 & -8.465 \\
\hline BAU 294-93×IR-36 & -7.583 & -13.731 & -29.956 & -35.599 & -23.875 & -25.536 \\
\hline BAU 294-93×BD-202 & -4.053 & -11.669 & -32.625 & -36.986 & -29.517 & -29.997 \\
\hline BAU 294-93×IR-64 & -17.834 & -20.981 & -36.797 & -38.031 & -22.980 & -27.334 \\
\hline BAU 295-93×IR-36 & -16.775 & -22.549 & -38.712 & -46.571 & -32.958 & -34.484 \\
\hline BAU 295-93×BD-202 & -14.276 & -21.318 & -25.483 & -33.923 & -12.925 & -13.427 \\
\hline BAU 295-93×IR-64 & -29.455 & -31.947 & -39.890 & -40.899 & -13.982 & -18.764 \\
\hline BAU 213-92×IR-36 & 4.280 & 1.336 & $3.295^{* *}$ & $2.989^{*}$ & -1.057 & -2.024 \\
\hline BAU 213-92×BD-202 & $11.195^{* *}$ & $9.071^{* *}$ & $4.134^{* *}$ & $6.923^{* *}$ & -5.883 & -7.650 \\
\hline BAU 213-92×IR-64 & -6.287 & -17.765 & -16.305 & -25.463 & -10.051 & -16.103 \\
\hline
\end{tabular}




\begin{tabular}{|c|c|c|c|c|c|c|}
\hline BAU 200-90×IR-36 & -9.251 & -14.546 & -9.904 & -14.921 & 2.165 & 0.133 \\
\hline BAU $200-90 \times$ BD-202 & 5.450 & -2.083 & -4.231 & -7.961 & -6.592 & -10.977 \\
\hline BAU 200-90×IR-64 & -9.036 & -13.313 & -20.306 & -23.653 & -10.083 & -18.411 \\
\hline BAU 205-90×IR-36 & 1.062 & -13.049 & $3.116^{* *}$ & -23.077 & 2.755 & -1.098 \\
\hline BAU 205-90×BD-202 & $8.475^{* *}$ & -7.829 & $15.531^{* *}$ & -12.506 & $7.164^{* *}$ & $6.114^{* *}$ \\
\hline BAU 205-90×IR-64 & -5.236 & -9.950 & -9.882 & -20.199 & -5.364 & -9.277 \\
\hline BR-8×IR-36 & $21.679^{* *}$ & $19.547^{* *}$ & $27.573^{* *}$ & $17.773^{* *}$ & $4.730^{* *}$ & 2.343 \\
\hline BR- $8 \times$ BD-202 & $27.637^{* *}$ & $23.575^{* *}$ & $27.144^{* *}$ & $19.518^{* *}$ & -0.021 & -0.602 \\
\hline BR-8×IR-64 & $16.884^{* *}$ & $8.871^{* *}$ & $9.089^{* *}$ & 2.034 & -6.400 & -11.607 \\
\hline IR-36-2×IR-36 & $6.849^{*}$ & 0.899 & $8.517^{* *}$ & 0.365 & $3.860^{*}$ & 3.110 \\
\hline IR-36-2×BD-202 & $19.403^{* *}$ & $11.182^{* *}$ & $18.412^{* *}$ & $11.493^{* *}$ & -0.697 & -4.172 \\
\hline IR-36-2×IR-64 & $24.021^{* *}$ & $20.039^{* *}$ & $2.477^{*}$ & -3.254 & -16.802 & -23.613 \\
\hline BAU 211-90×IR-36 & $33.117^{* *}$ & $31.392^{* *}$ & $25.829^{* *}$ & $23.802^{* *}$ & -5.807 & -7.868 \\
\hline BAU 211-90×BD-202 & $31.299^{* *}$ & $27.702^{* *}$ & $24.670^{* *}$ & $25.014^{* *}$ & -4.694 & -9.347 \\
\hline BAU 211-90×IR-64 & 0.610 & -6.685 & -23.262 & -31.096 & -23.079 & -30.334 \\
\hline BAU 269-92×IR-36 & $16.675^{* *}$ & $6.896^{*}$ & $25.966^{* *}$ & $12.178^{* *}$ & $8.422^{* *}$ & $5.163^{* *}$ \\
\hline BAU 269-92×BD-202 & $5.785^{*}$ & -4.386 & -1.063 & -10.426 & -5.999 & -6.179 \\
\hline BAU 269-92×IR-64 & -8.677 & -8.778 & -16.561 & -15.823 & -8.458 & -12.900 \\
\hline BAU 275-92×IR-36 & -2.282 & -13.564 & -26.218 & -46.293 & -24.110 & -26.272 \\
\hline BAU 275-92×BD-202 & -1.117 & -13.661 & 28.157 & -46.870 & -25.117 & -25.136 \\
\hline BAU 275-92×IR-64 & -11.784 & -13.508 & -38.456 & -46.395 & -30.262 & -33.762 \\
\hline $\operatorname{SEm} \pm$ & 1.970 & 2.280 & 0.780 & 0.900 & 1.220 & 1.410 \\
\hline $\mathrm{CD}(p=0.05)$ & 5.460 & 6.310 & 2.160 & 2.490 & 3.380 & 3.900 \\
\hline $\mathrm{CD}(p=0.01)$ & 7.180 & 8.290 & 2.830 & 3.270 & 4.440 & 5.130 \\
\hline
\end{tabular}

heterosis for test weight was found in BAU-275-92×IR-36 and BAU-275-92 $\times$ BD-202 and desirable heterobeltiosis was shown by the cross i.e. IR-36-2×IR-36, IR-36- $2 \times$ BD-202 and BAU 198-90×IR-36. Similar results were obtained by Julfiquar and Tepora (1992) and Reddy and Nerker (1995). Significant heterosis over mid parent was found in BAU-211-90 $\times \mathrm{BD}-$ 202, BAU-211-90 $\times$ IR-36, BR- $8 \times$ BD-202 whereas over better parent BAU-270-92 $\times$ BD-202 exhibited better heterosis for the character total number to tillers plant ${ }^{-1}$. The significant heterosis over mid parent was exhibited by BAU-211-90×IR36 and BR- $8 \times$ BD-202 and over better parent was shown by BR- $8 \times$ BD-202 and BAU-270-92 $\times$ BD-202 for total number of effective tillers plant ${ }^{-1}$ which can be further exploited. Similar are the results of works of many other scientists (Pandey et al., 1995; Reddy and Nerker, 1995 and Singh and Haque, 1999). For panicle length significant heterosis over mid parents and better parents in desired magnitude was found in BAU-21392 $\times$ IR-36 and BAU-291-93 $\times$ IR-64 which is in accordance with other studies (Julfiquar and Tepora, 1992 and Pandey et al., 1995). The cross BAU-291-93×IR-36 and BAU-211$90 \times$ IR-36 had shown marked heterosis over both mid parent and better parent for biological yield which is similar to the findings of Quin et al. (1995). For the character number of grains panicle ${ }^{-1}$, single cross i.e. BAU-29-93 $\times$ IR-64 had shown

significant relative heterosis and heterobeltiosis similar to the studies of Dong et al. (1995) and Rao et al. (1996). None of the crosses exhibited significant heterosis for spikelet fertility. This result was also obtained by Rao et al. (1996). The crosses i.e. BAU-274-92 $\times$ IR-36 followed by BAU-269$92 \times$ IR-36 and BAU-205-90 $\times$ BD-202 had shown significant heterosis over mid parent for harvest index and the crosses i.e. BAU-205-90×BD-202, BAU-274-92 $\times$ IR-36 and BAU269-92 $\times$ IR-36 exhibited better heterosis over better parent. Similar information was also gathered by Vishwakarma et al. (1999), Jelodar et al. (2010), Mirarab et al. (2011), Vennila et al. (2011) and Zhou et al. (2014).

The inheritance of yield and most of the yield contributing characters is polygenic in nature which shows continuous variation. The choice of appropriate breeding procedure depends on the type of gene action involved in the expression of these characters (Table 4) in a genetic population. Study of the nature gene action involved reveals that the sca variances are higher than gca variances for all the characters thus showing the preponderance of non-additive gene action (dominance and epistasis) and therefore, heterosis breeding may be rewarding.

There is continuous discussion on the validity of the causes that (C) 2015 PP House 


\begin{tabular}{|c|c|c|}
\hline Characters & $\begin{array}{l}\text { Best hybrids on } \\
\text { the basis of per se } \\
\text { performance }\end{array}$ & $\begin{array}{c}\text { Best specific } \\
\text { combiners }\end{array}$ \\
\hline \multirow{5}{*}{$\begin{array}{l}\text { Days of } \\
\text { panicle } \\
\text { emergence }\end{array}$} & Mashuri×IR -36 & Mashuri×IR-36 \\
\hline & BAU-295-93×IR-36 & BAU $270-92 \times$ BD-202 \\
\hline & BAU-295-93×IR-64 & BAU 291-93×IR-64 \\
\hline & BAU-269 -92×IR-36 & BAU 213-92×IR-36 \\
\hline & BAU-269-92×IR-64 & BAU 211-90×IR-64 \\
\hline \multirow{5}{*}{$\begin{array}{l}\text { Plant } \\
\text { height } \\
(\mathrm{Cm})\end{array}$} & BAU-198-90×IR-64 & BAU 198-90×IR-64 \\
\hline & Mashuri×IR-64 & BAU 274-92×IR-64 \\
\hline & BAU-274 $-92 \times$ BD -202 & BAU $295-93 \times$ BD-202 \\
\hline & BR- $8 \times$ BD-202 & BR- $8 \times$ IR-36 \\
\hline & BR-8×IR -64 & BR- $8 \times$ BD-202 \\
\hline \multirow{8}{*}{$\begin{array}{l}\text { Days to } \\
\text { maturity }\end{array}$} & BAU-198-90×IR-64 & BAU 213-92×IR-36 \\
\hline & Mashuri×IR-36 & BAU $270-92 \times$ BD-202 \\
\hline & BAU-294-93×IR-64 & BAU 294-93×IR-64 \\
\hline & BAU-295-93×IR-36 & BAU 274-92×IR-64 \\
\hline & BAU-200-90×IR-36 & BAU $274-92 \times$ BD-202 \\
\hline & BAU-269-92×IR-36 & Mashuri×IR-36 \\
\hline & BAU-269-92×IR-64 & BAU 211-90×IR-64 \\
\hline & BAU-275-92×IR-64 & \\
\hline \multirow{3}{*}{$\begin{array}{l}\text { Total } \\
\text { number } \\
\text { of leaves } \\
\text { plant }^{-1}\end{array}$} & BAU-205-90×BD-202 & \\
\hline & BAU-211-90×IR-36 & \\
\hline & BAU-291-93×IR-36 & \\
\hline \multirow{5}{*}{$\begin{array}{l}\text { Flag leaf } \\
\text { area }\end{array}$} & BAU-291-93×IR-64 & BAU 198-90×IR-64 \\
\hline & BAU-294-93×IR-64 & Mashuri×IR-36 \\
\hline & BAU-295-93×IR-64 & BAU 274-92×IR-36 \\
\hline & BAU-205-90×IR-36 & IR-36-2×IR-64 \\
\hline & IR-36-2×IR-64 & \\
\hline \multirow{5}{*}{$\begin{array}{l}\text { Total } \\
\text { number } \\
\text { of tillers } \\
\text { plant }^{-1}\end{array}$} & BAU-270-92×BD-202 & \\
\hline & BAU-205-90×BD-202 & \\
\hline & BR- $8 \times$ BD-202 & \\
\hline & BAU-211-90×IR-36 & \\
\hline & BAU-211-90×BD-202 & \\
\hline \multirow{10}{*}{$\begin{array}{l}\text { Total } \\
\text { number } \\
\text { of } \\
\text { effective } \\
\text { tillers } \\
\text { plant }^{-1}\end{array}$} & BAU-270-92×BD-202 & \\
\hline & BAU-205-90×BD-202 & \\
\hline & BR- $8 \times$ BD-202 & \\
\hline & IR-36-2×BD-202 & \\
\hline & BAU-211 -90×IR-36 & \\
\hline & BAU-211-90×BD-202 & \\
\hline & BAU-198-90×IR-36 & \\
\hline & BAU-291-93×IR-64 & \\
\hline & BAU-213-92×IR-36 & \\
\hline & IR-36-2×IR-64 & \\
\hline
\end{tabular}

\begin{tabular}{|c|c|c|}
\hline $\begin{array}{l}\text { No. of } \\
\text { grains } \\
\text { panicle } \\
\end{array}$ & BAU -291-93×IR-64 & $\begin{array}{c}\text { Mashuri } \times \text { IR-36 } \\
\text { IR-36-2×BD-202 }\end{array}$ \\
\hline $\begin{array}{l}\text { Grain } \\
\text { yield } \\
\text { panicle }^{-1}\end{array}$ & $\begin{array}{l}\text { BAU-198-90×IR-36 } \\
\text { Mashuri } \times \text { IR-36 } \\
\text { BAU-274-92×IR-36 } \\
\text { BAU-274-92×IR-64 } \\
\text { BAU-211-90×IR-36 } \\
\text { BAU-211-90×IR-64 }\end{array}$ & \\
\hline $\begin{array}{l}1000 \\
\text { grain } \\
\text { weight }\end{array}$ & $\begin{array}{c}\text { BAU-198-90×IR-36 } \\
\text { BAU-270-92×IR-36 } \\
\text { IR-36-2×IR-36 } \\
\text { IR-36-2×BD-202 } \\
\text { BAU-211-90×BD-202 } \\
\text { BAU-275-92×IR-36 } \\
\text { BAU-275-92×IR-202 } \\
\text { BAU-275-92×IR-64 }\end{array}$ & $\begin{array}{c}\text { BAU } 294-93 \times \text { IR-64 } \\
\text { BAU 200-90×BD-202 } \\
\text { BAU 200-90×IR-64 } \\
\text { IR-36-2×IR-36 } \\
\text { IR-36-2×IR-64 }\end{array}$ \\
\hline $\begin{array}{l}\text { Biologi- } \\
\text { cal yield }\end{array}$ & 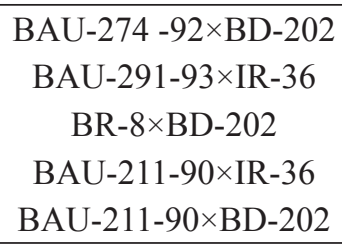 & $\begin{array}{c}\text { Mashuri } \times \text { BD-202 } \\
\text { BAU 295-93×IR-36 } \\
\text { BR-8 } \times \text { IR-64 } \\
\text { BAU 269-92×IR-64 }\end{array}$ \\
\hline $\begin{array}{l}\text { Grain } \\
\text { yield } \\
\text { plant }^{-1}\end{array}$ & $\begin{array}{c}\text { BAU-274-92×IR-36 } \\
\text { BR-8×IR-36 } \\
\text { BR-8×BD-202 } \\
\text { BAU-211-90×IR-36 } \\
\text { BAU-211-90×BD-202 } \\
\text { BAU-269-92×IR-36 }\end{array}$ & $\begin{array}{c}\text { Mashuri } \times \text { IR-36 } \\
\text { Mashuri } \times \text { BD-202 } \\
\text { BAU } 291-93 \times \text { BD-202 } \\
\text { BAU } 205-90 \times \text { IR-36 } \\
\text { BR-8 } \times \text { IR-64 }\end{array}$ \\
\hline $\begin{array}{l}\text { Harvest } \\
\text { index }\end{array}$ & $\begin{array}{c}\text { Mashuri } \times \text { IR-36 } \\
\text { BAU-274-92×IR-36 } \\
\text { BAU-205-90×BD-202 } \\
\text { BR-8×IR-36 } \\
\text { BAU-269-92×IR-36 }\end{array}$ & $\begin{array}{c}\text { Mashuri } \times \text { IR-36 } \\
\text { BAU 294-93×BD-202 } \\
\text { BAU 213-92×IR-36 } \\
\text { BAU 200-90×IR-36 } \\
\text { BAU 205-90×IR-36 } \\
\text { IR-36-2×IR-36 } \\
\text { IR-36-2×BD-202 }\end{array}$ \\
\hline
\end{tabular}

are responsible for heterosis. Over the time depending on the specific study and the crop, epistasis has been identified as part of the control of heterosis, mainly in rice. This was especially true for complex characters such as yield. For example, Yu et al. (1997) found in rice that dominance and overdominance had little effect on heterosis for yield and its components, while epistasis was found to have a major effect in this respect. Epistasis was found to be important in rice heterosis also in the control of simpler traits such as heading date and plant height (Yu et al., 2002). In their theoretical framework for the resolution of epistasis, dominance, and overdominance effects on heterosis, Melchinger et al. (2007) referred to the epistasis effect by the term 'augmented dominance effect. Birchler et al. 
(2010) tended to conclude that where the effect of these three gene actions is considered, the relative role of each might be related to the trait in question.

\section{Conclusion}

BAU-274-92 $\times$ IR-36 gave significantly high positive heterosis followed by BR- $8 \times$ IR-36, BR- $8 \times$ BD-202, BAU-211-90 $\times$ IR36 , BAU-211 $\times$ BD-202 and BAU-269-92 $\times$ IR-36 for grain yield plant -1 . The gca status of the parents revealed that high $\times$ high, high $\times$ low and low $\times$ low combinations shows involvement of both additive and non-additive gene effects. These cross combinations can be further improved through simple selection procedures (high $\times$ high). Other combinations with high $\times$ low gca can be further improved through transgression. The combinations with low $\times$ low gca can be utilized for hybrid development through CGMS system.

\section{Future Thrust}

The crosses viz. BAU-274-92 $\times$ IR-36, BR- $8 \times$ BD-202, BAU211-90×IR-36, BAU-211×BD-202, and BAU-269-92 $\times$ IR36 can be more beneficial in future if handled carefully and intensive selection pressure can be applied to isolate transgressive segregants and/or can be utilised for development of high yielding hybrids if combined with suitable CGMS lines suitable for (drought/rainfed) environment.

\section{References}

Ali, S.S., Khan, M.G., 1995. Studies for heterosis and combining ability in rice. Pakistan Journal of Scientific and Industrial Research 38(5-6), 200-204.

Birchler, J.A., Yao, H., Chudalayandi, S., Vaiman, D., Veitia, R.A., 2010. Heterosis. The Plant Cell 22, 2105-2112.

Dong Xu, Z., Fenghao, D., Zhen, Z., Zhang, Xue Quing, Y., 1995. Heterosis of hybrid rice combined with indica photoperiod temperature sensitive genitive male sterile line M25 with marker. Acta Agricultural Zhejiangensis 7(4), $232-325$.

FAO, 2012. World Agriculture towards 2030/2050: The 2012 Revision. Available from http://www.fao.org/docrep/016/ ap106e/ap106e.pdf

Fehr, W.R., 1987. Heterosis In: Principles of cultivar development: Theory and Techniques (Vol. 1). Macmillan Publishing Company, New York, pp 115.

Fujimura, T., Akagi, H., Oka, M., Nakamura, A., Sawada, R., (1996). Establishment of a rice protoplast culture and application of an asymmetric protoplast fusion technique to hybrid rice breeding. Plant Tissue Cult. Lett. 13, 243-247.

Jelodar, N.B., 2010. Heterosis and combining ability analysis for yield and related-yield traits in hybrid rice.
International Journal of Biology 2(2), 222- 231.

Julfiquar, A.W., Tepora, N.M., 1992. Heterosis in some quantitative characters in $\mathrm{F} 1$ hybrid rice (Oryza sativa L.). CLSU Scientific Journal of Philippines 12(2), 30-36.

Kampthorne, O., 1957. An introduction to Genetic Statistics. John Wiley and Sons. Inc., New York, Chapman and Hall, London.

Mackill, D.J., Lei, X.M., (1997). Genetic variation for traits related to temperate adaptation of rice cultivars. Crop Sci. 37, 1340-1346.

Melchinger, A.E., Utz, H.F., Piepho, H.P., Zeng, Z.B., Schon, C.C., 2007. The role of epistasis in the manifestation of heterosis: a systemsoriented approach. Genetics 177, 1815-1825.

Miller, B.C., Foin, T.C., Hill, J.E., (1993). CARICE: a rice model for scheduling and evaluating management actions. Agron. J. 85, 938-947.

Mirarab, M., Asadollah, A., Mohamad, H.P., 2011. Study on combining ability, heterosis and genetic parameters of yield traits in rice. African Journal of Biotechnology 10(59), 12512-12519.

Nemoto, K., Morita, S., Baba, T., (1995). Shoot and root development in rice related to the phyllochron. Crop Sci. 35, 24-29.

Pandey, M.P., Singh, J.P., Singh, H., 1995. Heterosis breeding for grain yield and other agronomic characters in rice (Oryza sativa L.). Indian Journal of Genetics 55(4), 438-455.

Paterson, A.H., Freeling, M., Sasaki, T., (2005). Grains of knowledge: genomics of model cereals. Genome Res. 15, 1643-1650.

Pratap, N., Shekhar, R., Singh, P.K., Soni, S.K., 2013. Combining ability, gene action and heterosis using CMS lines in hybrids rice (Oryza sativa L.). The Bioscan 8(4), 1521-1528.

Quin, Y., Quan, L.Y., Zhang, S.Z., Yulan, W., Wang, Y.B., Zhang, G.Q., 1995. Analysis of heterosis combining ability and path coefficients in japonica rice hybrid. Acta Agriculture Scanghai 11(4), 23-27.

Rao, A.M., Ramesh, S., Kulkarni, R.S., Savithramma, D.L., Madhusudan, K., 1996. Heterosis and combining ability in rice. Crop Improvement 23(1), 23-56.

Reddy, C.D.R., Nerkar, Y.S., 1995. Heterosis and inbreeding depression in upland rice crosses. Indian Journal of Genetics 55(4), 389-393.

Reddy, J.N., De, R.N., 1996. Genetic variability in lowland rice. Madras Agricultural Journal 83(4), 269-270.

Saidaiah, P., Sudheer K.S., Ramesha, M. S., 2010. Combining ability studies for development of new hybrids in rice over environments. Journal of Agricultural Science 2(2), 225-233.

Singh, M., Maurya, D.M., 1999. Heterosis and inbreeding 
depression in rice for yield and yield components using CMS system. Oryza, 36(1), 24-27.

Singh, S.K., Haque, M.F., 1999. Heterosis for yield and components in rice (Oryza sativa L.). Indian Journal of Genetics 52(2), 237-238.

Thakare, I.S., Patel, A.L., Mehta, A.M., 2013. Line×Tester analysis using CMS system in rice (Oryza sativa L.). The Bioscan 8(4), 1379-1381.

Vanaja, T., Babu, L.C., 2004. Heterosis for yield and yield components in rice (Oryza sativa L.). Journal of Tropical Agriculture 42(1/2), 43-44.

Vennila, S., Anbuselvam, Y., Palaniraja, K., 2011. Heterosis studies for yield and its components in rice (Oryza sativa L.). International Journal of Recent Scientific Research 2(9), 261-262.

Vishwakarma, D.N., Maurya, D.M., Verma, G.P., Vishwakarma, S.R., 1999. Heterosis for yield components in rice hybrids (Oryza sativa L.). Indian Journal of Agricultural Sciences 69(7), 530.

Wayne, S.C., Dilday, R.H., (2003). Rice: Origin, History, Technology, and Production. Wiley Series in Crop Science, John Wiley\&Sons, Inc. p. 324.

Xiong, L.W., Yi, L., Tingchai, Y., 1996. The heterotic effects on dry matter production and grain yield formation in hybrid rice. Journal of Fujjan Agricultural University 25(3), 260-265.

Yolanda, J.L., Das, L.D.V., 1996. Heterosis in hybrid rice. Madras Agricultural Journal 83(2), 115-117.

Yuan, L.P., 1994. Increasing yield potential in rice by exploitation of heterosis. In: Virmanni SS (ed) Hybrid rice technology. New developments and future prospects. IRRI, Manila, Philippines, p. 1-6.

Yu, S.B., Li, J.X., Xu, C.G., Tan, Y.F., Gao, Y.J., Li, X.H., Zhang, Q., Saghai Maroof, M.A., 1997. Importance of epistasis as the genetic basis of heterosis in an elite rice hybrid. Proceedings of the National Academy of Sciences, USA 94, 9226-9231.

Yu, S.B., Li, J.X., Xu, C.G., Tan, Y.F., Li, X.H., Zhang, Q., 2002. Identification of quantitative trait loci and epistatic interactions for plant height andheading date in rice. Theoretical and Applied Genetics 104, 619-625.

Zhou, G., Chen, Y., Yao, W., Zhang, C., Xie, W., Hua1, J., Xing, Y., Xiao, J., Zhang, Q., 2014. Genetic composition of yield heterosis in an elite rice hybrid. PNAS 109(39), 15847-15852. 